\title{
In vivo imaging through the entire thickness of human cornea by full- field optical coherence tomography
}

Viacheslav Mazlin, Peng Xiao, Eugénie Dalimier, Kate Grieve, Kristina Irsch, et al.

Viacheslav Mazlin, Peng Xiao, Eugénie Dalimier, Kate Grieve, Kristina Irsch, José Sahel, Mathias Fink, Claude Boccara, "In vivo imaging through the entire thickness of human cornea by full-field optical coherence tomography," Proc. SPIE 10474, Ophthalmic Technologies XXVIII, 104740 S (19 February 2018); doi: 10.1117/12.2288947

SPIE. Event: SPIE BiOS, 2018, San Francisco, California, United States 


\title{
In vivo imaging through the entire thickness of human cornea by full-field optical coherence tomography
}

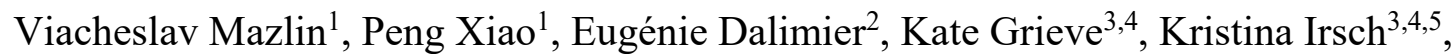 \\ José Sahel ${ }^{3,4,6}$, Mathias Fink ${ }^{1}$, and Claude Boccara ${ }^{1,2}$
}

\begin{abstract}
${ }^{1}$ Institute Langevin, ESPCI Paris, PSL Research University, Paris, France; ${ }^{2}$ LLTech SAS, Paris, France; ${ }^{3}$ Vision Institute/CIC 1423, UPMC-Sorbonne Universities, UMR S 968 / INSERM, U968 / CNRS, UMR 7210; ${ }^{4}$ Quinze-Vingts National Eye Hospital, Paris, France; ${ }^{5}$ Laboratory of Ophthalmic Instrument Development, The Wilmer Eye Institute, The Johns Hopkins University School of Medicine, Baltimore, Maryland, USA; ${ }^{6}$ University of Pittsburgh School of Medicine, Pittsburgh, USA.
\end{abstract}

\begin{abstract}
Despite obvious improvements in visualization of the in vivo cornea through the faster imaging speeds and higher axial resolutions, cellular imaging stays unresolvable task for OCT, as en face viewing with a high lateral resolution is required. The latter is possible with FFOCT, a method that relies on a camera, moderate numerical aperture (NA) objectives and an incoherent light source to provide en face images with a micrometer-level resolution. Recently, we for the first time demonstrated the ability of FFOCT to capture images from the in vivo human cornea ${ }^{1}$. In the current paper we present an extensive study of appearance of healthy in vivo human corneas under FFOCT examination. En face corneal images with a micrometer-level resolution were obtained from the three healthy subjects. For each subject it was possible to acquire images through the entire corneal depth and visualize the epithelium structures, Bowman's layer, sub-basal nerve plexus (SNP) fibers, anterior, middle and posterior stroma, endothelial cells with nuclei. Dimensions and densities of the structures visible with FFOCT, are in agreement with those seen by other cornea imaging methods. Cellular-level details in the images obtained together with the relatively large field-of-view (FOV) and contactless way of imaging make this device a promising candidate for becoming a new tool in ophthalmological diagnostics.
\end{abstract}

Keywords: Optical coherence tomography, Imaging systems, Ophthalmic devices, Human eye imaging, Cornea imaging, In vivo imaging, Cornea, Full-field optical coherence tomography.

\section{INTRODUCTION}

Visualization of cells and other micrometer-size objects in the patient's cornea opens a possibility for earlier and more accurate diagnosis of the corneal diseases. Currently, confocal microscope, given its ability of en face viewing with a micrometer-level resolution ${ }^{2,3}$, is the only clinical tool capable to see these features through the entire corneal depth. Unfortunately, confocal microscopy is not exempt from drawbacks, being the relatively small field-of-view and typical requirement of contact with an eye. Another imaging technique, optical coherence tomography (OCT) ${ }^{4}$, has also been applied for in vivo human cornea imaging ${ }^{5,6}$. Despite clear successes in increasing the axial resolution and the imaging speed $^{7-11}$, up to now, visualization of micrometer-size cells, nuclei, nerves and other corneal objects throughout the whole corneal depth stayed unattainable task for OCT, as en face viewing with a high lateral resolution is required. The latter is possible with full-field OCT (FFOCT) ${ }^{12-13}$, a variation of OCT, which uses a camera to acquire 2D images directly, without a pixel-by-pixel scanning. Contrary to the conventional OCT, which acquires cross-sectional image containing information from the multiple depths inside the sample, FFOCT captures en face image from a single depth at a time. As a result, small depth of focus (DOF) microscope objectives with a moderate numerical aperture (NA) can be used in FFOCT, enabling non-contact imaging with a relatively large field-of-view (FOV) and a micrometer-level resolution. This resolution is not degraded by the speckle, because the incoherent light source is used. Additionally, resolution in FFOCT is not lowered by the geometrical aberrations ${ }^{14}$. FFOCT has been shown in a variety of applications, including imaging of biopsies of various tissues, studies in developmental biology, in vivo endoscopy, material characterization ${ }^{13}$, internal fingerprint imaging ${ }^{15}$, characterization of various ex vivo tissues from the anterior and posterior segments of animal eyes ${ }^{16}$ and in vivo imaging of the anterior segment of a rat ${ }^{17}$. Recently, we for the first time demonstrated the ability of FFOCT to capture images from

Ophthalmic Technologies XXVIII, edited by Fabrice Manns, Per G. Söderberg, Arthur Ho, Proc. of SPIE

Vol. 10474, 104740S · C 2018 SPIE · CCC code: 1605-7422/18/\$18 · doi: 10.1117/12.2288947 
the in vivo human cornea ${ }^{1}$. In the current paper we present an extensive study on appearance of healthy in vivo human corneas under FFOCT examination. En face corneal images with a micrometer-level resolution were obtained from the three healthy subjects. For each subject it was possible to acquire images through the entire corneal depth and visualize the epithelium structures, Bowman's layer, sub-basal nerve plexus (SNP) fibers, anterior, middle and posterior stroma, endothelial cells with nuclei. Dimensions and densities of the corneal structures, visible with FFOCT, were measured and compared with those, seen with other cornea imaging techniques. Detailed description of the device is provided.

\section{EXPERIMENTAL DEVICE AND METHODS}

\subsection{Description of FFOCT imaging system}

Detailed description of the device and the method is available in work ${ }^{1}$. A schematic drawing of the FFOCT instrument is presented in Figure 1. The instrument is based on a microscope in a Linnik interferometric configuration, typically used for FFOCT imaging. Illumination is provided by a $850 \mathrm{~nm}$ light-emitting diode (LED) source (model M850LP1, Thorlabs, USA) with a $30 \mathrm{~nm}$ bandwidth, resulting in a theoretical axial resolution of $7.7 \mu \mathrm{m}$ in the cornea. In both sample and reference arms, identical NIR objectives are used with a 10X magnification and a $0.3 \mathrm{NA}$, providing a theoretical transverse resolution according to the Rayleigh criterion of $1.7 \mu \mathrm{m}$. This resolution was verified experimentally by measuring the visible diameters of the gold nanoparticles, imaged with our interferometer. FOV provided by the optics was $1.26 \mathrm{~mm} \mathrm{X}$ $1.26 \mathrm{~mm}$. We used a high full well capacity CMOS camera (model Q-2A750-CXP, Adimec, Netherlands). A custom modification of the commercial software (LLTech SAS, France ${ }^{18}$ ) enables synchronization of the camera, the piezoelectric translation stage (PZT) and the LED. Images are displayed in real-time thanks to the rapid image processing.

\subsection{Imaging procedure}

According to the FFOCT imaging principle ${ }^{13}$, as the PZT is sinusoidally moving the mirror, synchronized image capture is taking place on the camera. The camera is triggered to take 2 images per modulation period of the piezo element. By means of a simple signal processing (image mutual subtraction, followed by the module) the 2 images are transformed into a single plane FFOCT image, which contains only the signal originating from the coherence volume. The single FFOCT image is captured in 3.4 milliseconds. Fast software processing scheme, implemented in our device, allows to capture and save several consecutive images of the same coherence volume in a row without any pause. Signal-to-noise ratio (SNR) can be increased by performing an averaging of these images, preceded by the image registration algorithm ${ }^{19,20}$ to compensate for the eye movements in the en face plane.

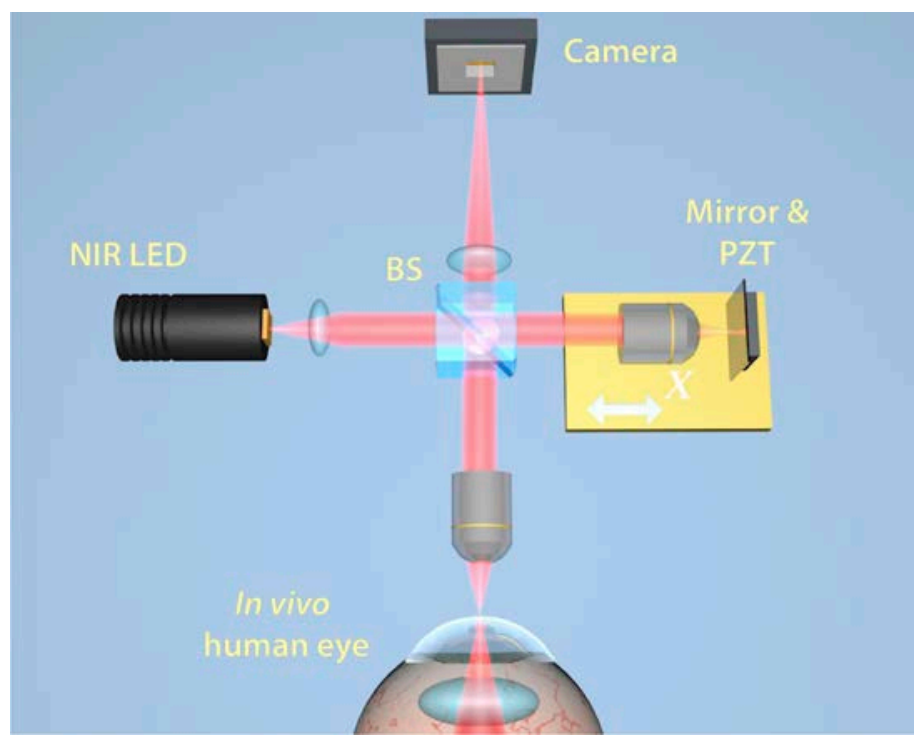

Fig. 1. Schematic of FFOCT in vivo imaging microscope. A motorized translation stage (yellow) can vary the optical path length of the reference arm by moving all of its elements (microscope objective, mirror and PZT) closer or farther from the beam splitter (BS). 
When imaging inside the cornea, the defocusing correction algorithm ${ }^{21}$, developed in our laboratory, was used to compensate for the difference between the air and corneal refractive indexes. More precisely, the deeper we intend to image inside the cornea, the more separated the focus and coherence planes become (due to the mismatch between the refractive indexes of air and cornea), and the more the whole reference arm of the device needs to be extended to match back the focus and coherence planes, therefore restoring the signal. This method is easy to implement for ex vivo imaging, however, is very complicated for in vivo imaging, as for every change in the eye position, the reference arm position has to be simultaneously changed, which is technically challenging. Yet, to obtain corneal images from each layer we developed a simple scheme, avoiding technical complications. Before the start of the imaging procedure, we select the corneal layer of interest and put the reference arm in the position suitable for imaging of this layer. Then, we move the whole interferometer closer and farther from the cornea, while keeping it approximately in the focus of the microscope objective. As soon as the layer of interest comes into the focus, the image of this layer appears on the display and gets manually saved.

The study was carried out on the three subjects (aged 20-35 years) with healthy corneas. Consent was obtained from each subject and the experimental procedures adhered to the tenets of the Declaration of Helsinki. During the experiment, subjects rested their chins and temples on a custom-built headrest, while looking at a fixation target within the device.

Eye was illuminated with the $850 \mathrm{~nm}$ wavelength light, typically used in clinical ophthalmic imaging. It is comfortably viewed by patients, due to the low sensitivity of the retina to NIR light, and poses no danger associated with photochemical damage. Retinal and corneal exposures were well below maximum permissible exposures (MPEs) specified by the ophthalmic safety standards (ANSI Z80.36-2016 and ISO 15004-2: 2007).

\section{RESULTS AND DISCUSSION}

Fig. 2 demonstrates FFOCT images from the anterior, middle and posterior parts of the in vivo cornea, obtained from the three subjects. Images frequently have round shaped profile, which reflects the fact that we are sectioning the spherical object with a plane (because the mirror in the reference arm is flat). For each of the three subjects in anterior part of the cornea we were able to visualize epithelium, SNP and anterior stromal keratocytes. Up to now, it was not possible to resolve the cells in the epithelium or distinguish between different epithelial layers, due to obscuring of the sub-surface signal by the strong tear film-cornea reflection on the surface, and due to low contrast between epithelial cells. In the SNP branching individual stromal nerves with thicknesses about 3 - $5 \mu \mathrm{m}$ were visible. Poorly reflecting layer between the SNP and stroma was the Bowman's layer. In the anterior stroma numerous bright egg-shaped keratocyte nuclei with diameters about $15 \mu \mathrm{m}$ were seen. In the middle stroma both the nuclei and the cell body of the keratocytes were visible. They were forming a dense network. In the posterior stroma keratocytes were prolonged comparing to the anterior and middle parts of the stroma. The above results are in agreement with current knowledge about the corneal structure ${ }^{22}$.

Table 1 provides comparison of keratocyte densities of the mentioned three corneal regions. Density was measured by counting the number of cell nuclei in the square area and dividing by that area.

Table 1. Measured normal stromal keratocyte densities

\begin{tabular}{|c|c|}
\hline Stromal part & $\begin{array}{c}\text { Cell density } \\
\text { (number of cells } / \mathbf{m m}^{\mathbf{2}} \text { ) }\end{array}$ \\
\hline Most anterior stroma & $29500 \pm 11450$ \\
\hline Middle stroma & $21750 \pm 6540$ \\
\hline Most posterior stroma & $23750 \pm 5350$ \\
\hline
\end{tabular}

Average cell density in the cornea was around 20000 cells $/ \mathrm{mm}^{2}$. The highest keratocyte concentrations was found in the anterior part adjacent to the Bowman's layer. The above is in agreement with the literature ${ }^{23}$. FFOCT provides a large FOV, as a result a cell density over the broader area can be measured, however it should be noted, that large FOV may affect accuracy of cell counting, as keratocytes layers located at different depths are present in a single image. 


\section{Subject \#}
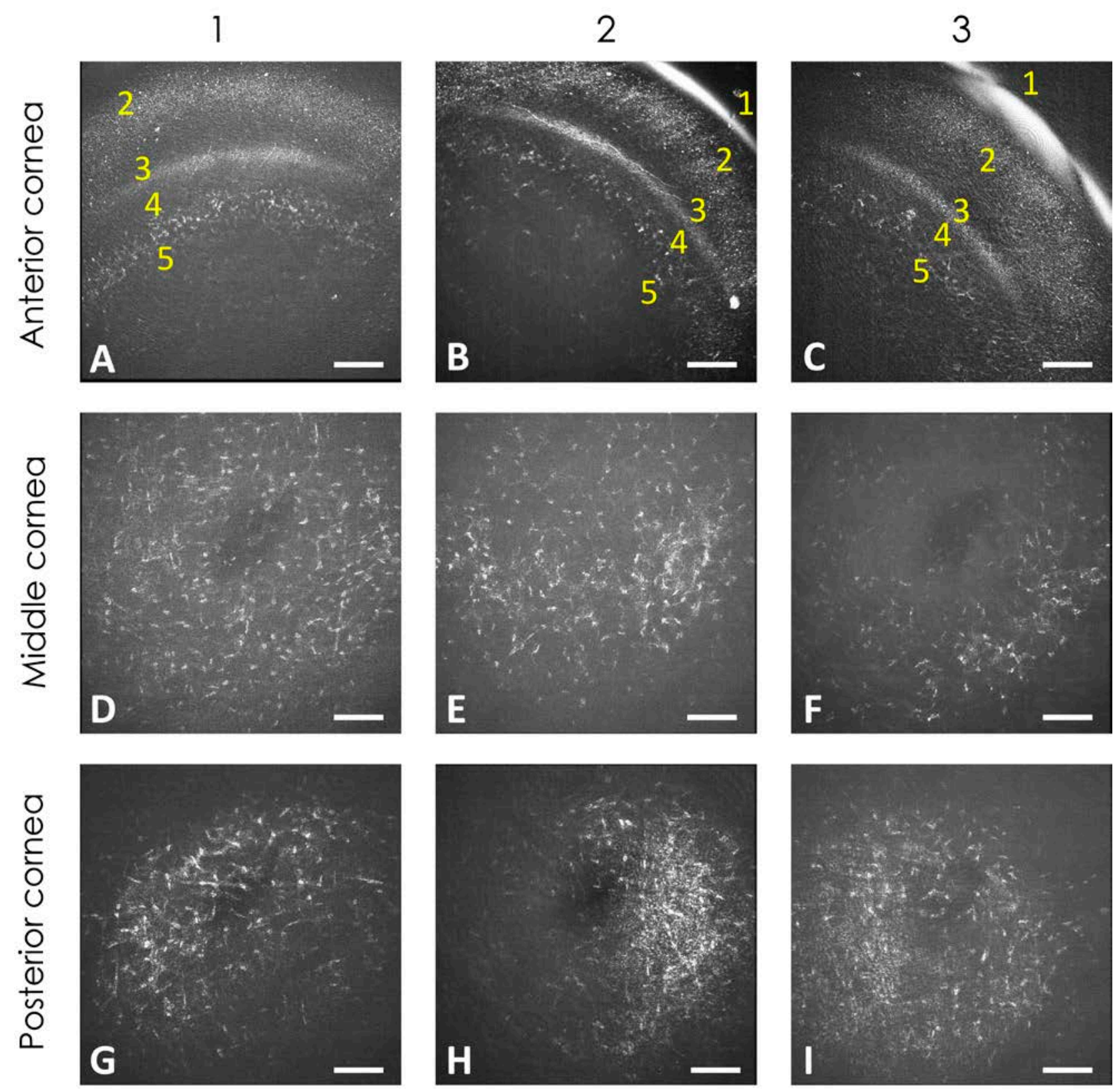

Fig. 2. Images of different corneal layers, obtained with FFOCT from three subjects. A, B, C - most anterior stroma; D, E, F - Middle stroma; G, H, I - most posterior stroma. 1 - bright reflection from the air - tear film interface; 2 - epithelium; 3 SNP with individual nerves visible; 4 - Bowman's layer; 5 - most anterior stroma. Central dark spot, present in several images is due to the saturation of the central camera region as a result of the strong reflection from the corneal apex. White line is $200 \mu \mathrm{m}$.

Fig. 3 shows FFOCT images of the endothelial cell layer, acquired from the three subjects. We were able to resolve the hexagonal epithelial cell mosaic with nuclei. The average diameters of the cells and nuclei were $20 \mu \mathrm{m}$ and $4-7 \mu \mathrm{m}$, respectively. Descemet's was seen as a poorly reflecting layer. Interference fringe artifacts present in the images may be removed according to the method demonstrated in ${ }^{1}$. 


\section{Subject \#}

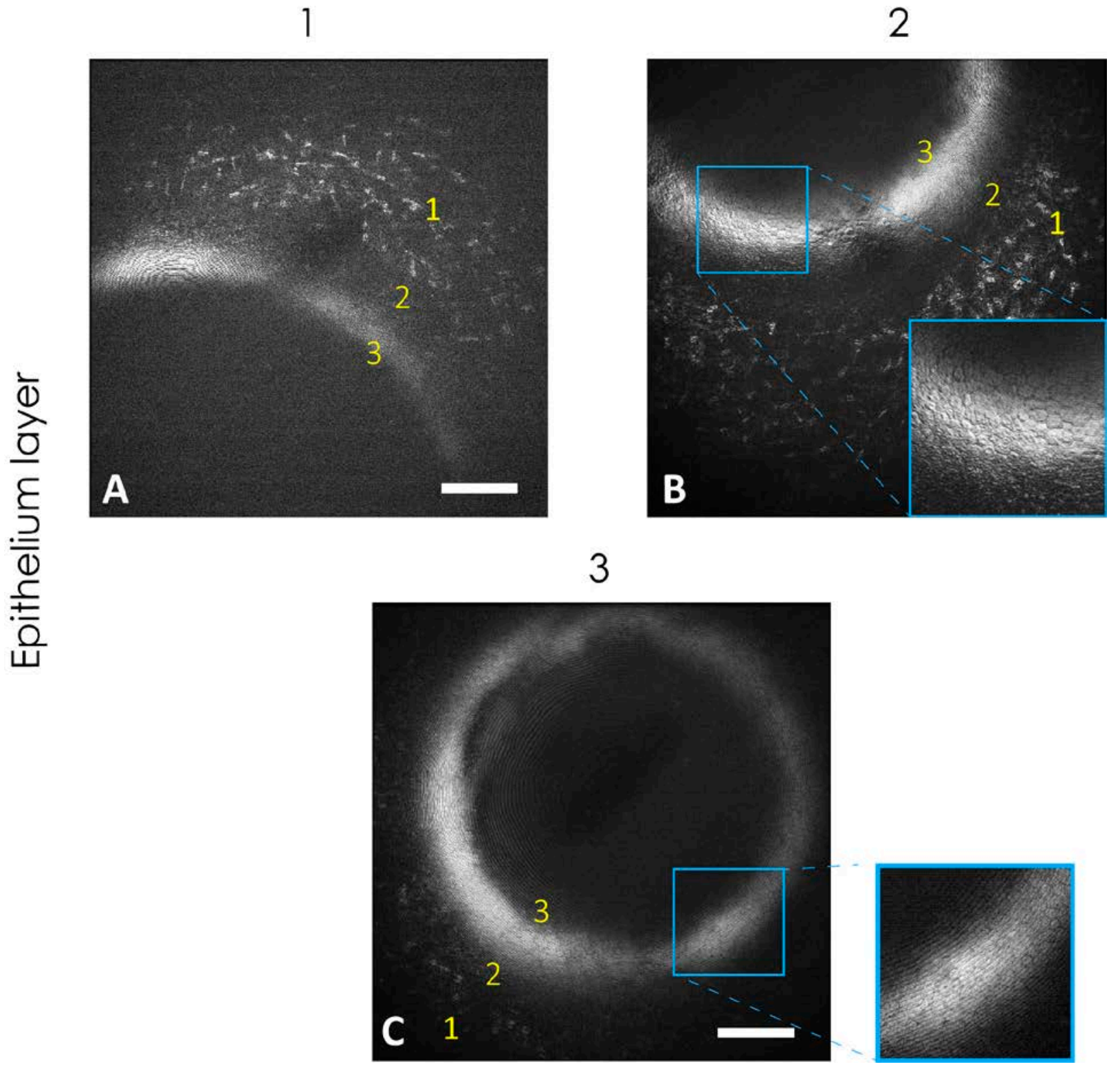

Fig. 3. FFOCT images of the endothelial cell layer, obtained from the three subjects. 1 - most posterior stroma; 2 Descemet's membrane; 3 - hexagonal endothelial cell mosaic with visible nuclei. Central dark spot, present in several images, is due to the saturation of the central camera region as a result of the strong reflection from the corneal apex. White line is $200 \mu \mathrm{m}$.

\section{CONCLUSION}

FFOCT images through the entire corneal depth were obtained from the three healthy subjects. Contactless operation together with the use of an NIR LED made the examination procedure comfortable for the subjects. All the images had a large FOV of $1.26 \times 1.26 \mathrm{~mm}^{2}$. We obtained images of epithelium, Bowman's layer, sub-basal nerve plexus (SNP), anterior, middle and posterior stroma, endothelial cells with visible nuclei. The measured nerve thicknesses, cell and nuclei diameters, cell densities were in agreement with the literature. Large FOV of FFOCT is potentially interesting for counting 
cell and nerve densities with a higher precision than is possible with the current techniques, however a new density measuring methodology, which would account for different corneal depths present in a single FFOCT image, would be required. Cellular-level details in images obtained together with the relatively large FOV and contactless way of imaging make this device a promising candidate for becoming a new tool in ophthalmological diagnostics.

\section{ACKNOWLEDGEMENTS}

This work was supported by HELMOLTZ Synergy grant funded by the European Research Council (ERC Synergy HELMHOLTZ, grant agreement \#610110).

\section{REFERENCES}

[1] Mazlin, V., Xiao, P., Dalimier, E., Grieve, K., Irsch, K., Sahel, J., Fink, M., Boccara, A.C., "In vivo high resolution human corneal imaging using full-field optical coherence tomography," Biomedical Optics Express (to be published).

[2] Stave, J., Zinser, G., Grümmer, G., Guthoff, R., "Der modifizierte Heidelberg-Retina-Tomograph HRT Erste Ergebnisse einer In-vivo-Darstellung von kornealen Strukturen," Der Ophthalmologe 99(4), 276-280 (2002).

[3] Guthoff, R., Zhivov, A., and Stachs, O., "In vivo confocal microscopy, an inner vision of the cornea - a major review," Clinical \& Experimental Ophthalmology 37, 100-117 (2009).

[4] Huang, D., Swanson, E., Lin, C., Schuman, J., Stinson, W., Chang, W., Hee, M., Flotte, T., Gregory, K. et al., "Optical coherence tomography," Science 254(5035), 1178-1181 (1991).

[5] Izatt, J., "Micrometer-Scale Resolution Imaging of the Anterior Eye In Vivo With Optical Coherence Tomography," Archives of Ophthalmology 112(12), 1584 (1994).

[6] Radhakrishnan, S., "Real-Time Optical Coherence Tomography of the Anterior Segment at $1310 \mathrm{~nm}$," Archives of Ophthalmology 119(8), 1179 (2001).

[7] Christopoulos, V., "In Vivo Corneal High-Speed, Ultra-High-Resolution Optical Coherence Tomography," Archives of Ophthalmology 125(8), 1027 (2007).

[8] Kaluzny, B., Kaluzny, J., Szkulmowska, A., Gorczynska, I., Szkulmowski, M., Bajraszewski, T., Targowski, P., Kowalczyk, A., "Spectral optical coherence tomography: a new imaging technique in contact lens practice," Ophthalmic and Physiological Optics 26(2), 127-132 (2006).

[9] Yadav, R., Lee, K., Rolland, J., Zavislan, J., Aquavella, J., Yoon, G., "Micrometer axial resolution OCT for corneal imaging," Biomedical Optics Express 2(11), 3037 (2011).

[10] Werkmeister, R., Sapeta, S., Schmidl, D., Garhöfer, G., Schmidinger, G., Aranha dos Santos, V., Aschinger, G., Baumgartner, I., Pircher, N. et al., "Ultrahigh-resolution OCT imaging of the human cornea," Biomedical Optics Express 8(2), 1221 (2017).

[11] Bizheva, K., Tan, B., MacLelan, B., Kralj, O., Hajialamdari, M., Hileeto, D., Sorbara, L., "Sub-micrometer axial resolution OCT for in-vivo imaging of the cellular structure of healthy and keratoconic human corneas," Biomedical Optics Express 8(2), 800 (2017).

[12] Beaurepaire, E., Boccara, A., Lebec, M., Blanchot, L., Saint-Jalmes, H., "Full-field optical coherence microscopy," Optics Letters 23(4), 244 (1998).

[13]Dubois, A., [Handbook Of Full-Field Optical Coherence Microscopy: Technology and Applications], Pan Stanford publishing (2016).

[14]Xiao, P., Fink, M., Boccara, A., "Full-field spatially incoherent illumination interferometry: a spatial resolution almost insensitive to aberrations," Optics Letters 41(17), 3920 (2016).

[15] Auksorius, E., Boccara, A., "Fingerprint imaging from the inside of a finger with full-field optical coherence tomography," Biomedical Optics Express 6(11), 4465 (2015).

[16] Grieve, K., Paques, M., Dubois, A., Sahel, J., Boccara, C., Le Gargasson, J., "Ocular Tissue Imaging Using Ultrahigh-Resolution, Full-Field Optical Coherence Tomography," Investigative Opthalmology \& Visual Science 45(11), 4126 (2004).

[17] Grieve, K., Dubois, A., Simonutti, M., Paques, M., Sahel, J., Le Gargasson, J., Boccara, C., "In vivo anterior segment imaging in the rat eye with high speed white light full-field optical coherence tomography," Optics Express 13(16), 6286 (2005).

[18] LLTech Imaging, http://www.lltechimaging.com (5 January, 2018). 
[19] Rueden, C., Eliceiri, K., "The ImageJ Ecosystem: An Open and Extensible Platform for Biomedical Image Analysis," Microscopy and Microanalysis 23(S1), 226-227 (2017).

[20]Tseng, Q., Duchemin-Pelletier, E., Deshiere, A., Balland, M., Guillou, H., Filhol, O., Thery, M., "Spatial organization of the extracellular matrix regulates cell-cell junction positioning," Proceedings of the National Academy of Sciences 109(5), 1506-1511 (2012).

[21] Labiau, S., David, G., Gigan, S., Boccara, A., "Defocus test and defocus correction in full-field optical coherence tomography," Optics Letters 34(10), 1576 (2009).

[22] Guthoff, R., Zhivov, A., Stachs, O., "In vivo confocal microscopy, an inner vision of the cornea - a major review," Clinical \& Experimental Ophthalmology 37(1), 100-117 (2009).

[23] Sanjay V. Patel, McLaren J.W., Hodge D.O., Bourne W.M., "Normal Human Keratocyte Density and Corneal Thickness Measurement by Using Confocal Microscopy In Vivo," Invest. Ophthalmol. Vis. Sci. 42(2), 333-339 (2001). 